Fig. 4. - Scleroderma venosum Boud. (2/3 de grandeur naturelle).

a. Coupe du mème individu.

b. Spores $\left(\frac{3+0}{1}\right)$.

Fi6. 5. - Mitrula sclerotipes Boud. (un peu grossi).
a. Le mème $\left(\begin{array}{l}3 \\ 1\end{array}\right)$.
$b$. Coupe d'un individu ( $\left.\frac{3}{1}\right)$.
$c$. Thèques et paraphyses $\left(\frac{280}{1}\right)$.
d. Spores $\left(\frac{3}{4} \underline{a}\right)$.

FIG. 6. - Ascobolus viridis Currey.
a. Le mème $\left(\frac{5}{1}\right)$.
$b$. Thèques et paraphyses $\left(\frac{80}{1}\right)$.
c. Spores $\left(\frac{3}{4} 0\right)$.
d. Opercule d'une thèque $\left(\frac{1}{1} \frac{1}{1}\right)$.

Fig. 7. - Ascobolus pusillus Boud. ( $\left.\frac{15}{1}\right)$.

a. Le mème, de grandeur naturelle.

$b$. Thèques et paraphyses $\left(\frac{100}{1}\right)$.

c. Spores $\left(\frac{3+0}{1}\right)$.

Fig. 8. - Spharia sepulta Boud. (aspect de grandeur naturelle).
a. Un échantillon séparé $\left(\frac{15}{1}\right)$.
$b$. Thèques $\left(\frac{100}{0}\right)$.
c. Spores $\left(\frac{320}{1}\right)$.

M. Cornu fait à la Société la communication suivante :

\title{
LISTE DES ESPECES RECUEILLIES LE 22 OCTOBRE PENDANT L'EXCURSION FAITE PAR LA SOCIÉTÉ DANS LA FORET DE SAINT-GERMAIN.
}

Cette excursion, que M. Aug. Michel avait bien voulu diriger, a permis de récoiter un assez grand nombre d'espèces, malgré l'extrême sécheresse du sol; seulement le nombre des échantillons fut très-restreint. De petites localités encore humides permirent néanmoins de recueillir quelques raretés.

Amanita Mappa.

- phalloides.

-- rubescens.

Armillaria mellea. CC.

Lepiota excoriata.

- granulosa.

- amiantina.

Tricholoma cinerascens.

Clitocybe infundibuliformis.

- laccata.

Collybia radicata.

- maculata.

- fusipes.

- butyracea.

- dryophila.
Collybia cirrata.

Mycena galericulata.

- rugosa.

- filopes.

- polygramma.

- parabolica.

- acicula.

- echinipes.

- capillaris.

Omphalia Fibula.

- hydrogramma.

- polyadelpha.

Pleurotus applicatus

Pholiota caperata.

- mutabilis. 
Hebeloma crustuliniforme.

- elatum.

Inocybe fastigiata.

- lacera.

- rimosa.

- geophylla.

- helomorpha.

- petiginosa.

Flammula carbonaria.

Naucoria escharoides.

Galera spicula.

- mycenopsis.

- hypnorum.

Tubaria furfuracea.

Psalliota arvensis.

Stropharia luteonitens.

Hypholoma fasciculare.

- sublateritium.

- appendiculatum.

Psathyra fibrillosa.

- disseminata.

Coprinus lagopus.

- exstinctorius.

- plicatilis.

Bolbitius hydrophilus.

Cortinarius castaneus.

- delibutus.

- hinnuleus.

- scululatus.

— isabellinus.

- paleaceus.

Hygrophorus discoideus.

Lactarius blennius.

- subdulcis.

- obnubilus.

- theiogalus.

Russula fragilis.

- cyanoxantha.

- chamæleontina.

- ochroleuca.

Marasmius urens.

- prasyosmus.

- epiphyllus.

- Rotula.

Panus stypticus (1).

Boletus scaber.

- subtomentosus.

Fistulina Hepatica

Polyporus fumosus.

- lucidus.
Polyporus perennis.

Dædalea quercina.

Merulius Corium.

Hydnum velutinum.

Irpex paradoxus.

- obliquus.

Radulum orbiculare.

Craterellus cornucopioides.

Thelephora sebacea.

Stereum spadiceum.

- hirsutum.

- purpureum.

Corticium comedens.

- evolvens.

- quercinum.

- carneum.

- læve.

Clavaria cristata.

- cinerea.

Tremella mesenterica.

- albida.

Dacrymyces deliquescens.

Helotium fructigenum.

Cenococcum geophilum.

Scleroderma vulgare.

Lycoperdon piriforme.

- gemmatum.

Cyathus striatus.

Xylaria polymorpha.

Hypoxylon udum.

- multiforme.

Eutypa lata.

Valsa stellulata.

Diatrype quercina.

Stictosphæeria Hoffmanri.

Dothidea Robertiani.

- graminum.

Pleospora Clavariorum.

Stilbum fimetarium.

Phacidium coronatum.

Coremium glaucocephalum.

Fusidium virens.

- griseum.

Sphæria macularis.

Sphærella acerina.

Stigmatea Urticæ.

Triblidium quercinum.

Chlorosplenium aruginosum.

Peziza aurantiaca.

- epidendri.

(1) Un des échantıllons prísentait une forme régulière avec n:ı stipe centrai. 
Peziza scutellata.

Peziza hyalina.

- nivea.

- cinerea.

- firma.

- Gouani.

Puccinia Glechoma.

- Rhinanthi.

Coleosporium Rhinanthacearum.

A la suite de cette communication, II. Roze fait part à la Société des craintes éprouvées par la Commission d'organisation au sujet du peu d'importance des résultats que paraissent devoir présenter les excursions annoncées par le programme de la Session comme devant être faites, le mercredi 24 octobre et le jeudi 25 , dans les bois de Meudon et d'Ecouen. A la demande de plusieurs personnes, il propose à la Société de modifier le programme de la façon suivante :

Mercredi 24 octobre. - Repos et visite dans l'après-midi aux collections paléontologiques et mycologiques dı Muséum d'histoire naturelle.

Jeudi 25 octobre. - Excursion dans la forêt de Montmorency, sous la direction de M. Boudier : cette excursion sera particulièrement consacrée à la récolte des Tubéracés.

Après une courte discussion, cette proposition est adoptée.

II. Cooke fait à la Société la communication suivante :

Sone allied species of fCidiacei, by M. C. CDOKE.

I desire to direct attention to two or three instances, out of a great many which have come under my notice, in which two very similar Fungi have been confounded together under the same specific name, or at least are so similar in many of their features as to be in danger of being mistaken the one for the other. The examples which I shall select are from the parasitic Coniomycetes, but it is not my intention to enter at all upon the question of the autonomy of these plants, on this occasion, as that would open a wide field for discussion. Suffice it to say that I am strongly of opinion that too much has been taken for granted, which should have been proved, and that many a pretty theory of dimorphism has no solid foundation, such as science has a right to demand.

The first example relates to the genus Peridermium, of which Peridermixm Pini is well known. Here however we have two forms, as intimated long ago by Link, the one on the twigs, the other on the leaves. Fuckel divided them as two distinct species under the names of P. Pini, and $P$. oblongisporum. I shall now aliude to them under the names proposed by Link, for the varieties, as $P$. corticolum and $P$. acicolum. There was no reason for Fuckel to invent a new name. 

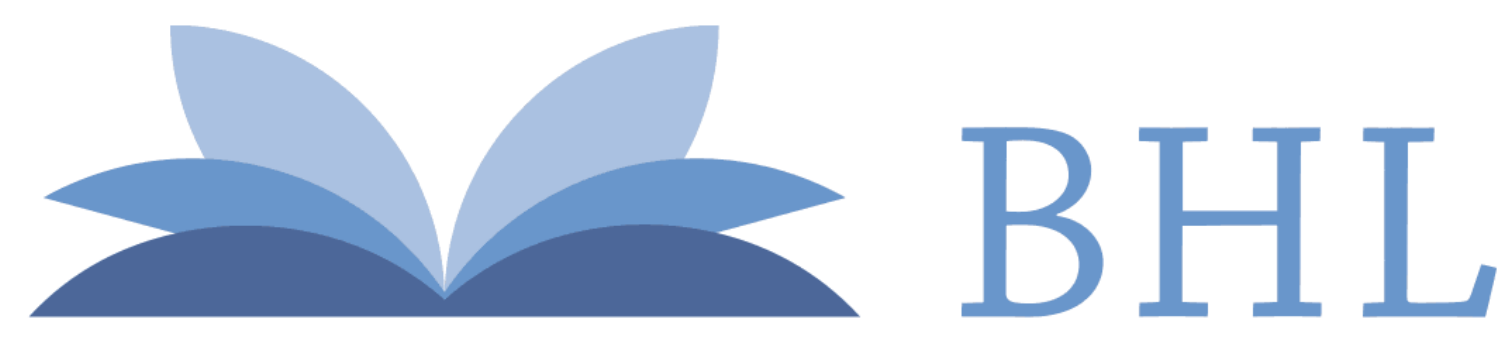

\section{Biodiversity Heritage Library}

Cornu, Maxime. 1877. "Liste Des Espèues Recueillies Le 22 Octobre Pendant L'Excursion Faite Par La Société Dans La Foret De Saint-Germain." Bulletin de la Société botanique de France 24, 312-314. https://doi.org/10.1080/00378941.1877.10830002.

View This Item Online: https://www.biodiversitylibrary.org/item/8647

DOI: https://doi.org/10.1080/00378941.1877.10830002

Permalink: https://www.biodiversitylibrary.org/partpdf/159545

\section{Holding Institution}

Missouri Botanical Garden, Peter H. Raven Library

\section{Sponsored by}

Missouri Botanical Garden

\section{Copyright \& Reuse}

Copyright Status: Public domain. The BHL considers that this work is no longer under copyright protection.

This document was created from content at the Biodiversity Heritage Library, the world's largest open access digital library for biodiversity literature and archives. Visit BHL at https://www.biodiversitylibrary.org. 